\title{
Old drug, new use: Can Amitriptyline improve mood and reduce skeletal muscle inflammation in a mouse model of Duchenne Muscular Dystrophy?
}

\author{
Jennifer Manning \\ Department of Physiology, UCC
}

\section{Introduction}

DMD is an inherited genetic disease affecting 1 in 3500 males. It results from the deletion of a gene which makes the protein Dystrophin. Dystrophin is essential for maintaining the structure of cells. In skeletal muscle, it acts as an anchor for structural components inside the cell. When dystrophin is missing, it leads to mechanical damage and tears in the muscle cell wall, which causes a cascade of events and leads to chronic inflammation.

The chemical components of this inflammatory response lead to extensive muscle damage in sufferers. Muscle tissue is replaced with collagen and fat cells, leading to a significantly weaker and dysfunctional muscle. Sufferers of DMD are wheelchair-bound by their teenage years, and don't normally live beyond their twenties. Clinical presentation of the disease occurs when young boys start walking; the weight put on the muscles of the calf exasperate the damage and they have difficulty running, jumping, they fall frequently or walk on their toes. It progresses quickly from here, with all weight-bearing muscles being affected. Other types of tissues in the body are affected by the loss of dystrophin too. Among them, weakened respiratory muscles lead to sufferers becoming dependant on ventilators. Eventually, the cardiac muscle of the heart is damaged. DMD is a progressive, debilitating and ultimately fatal disease.

Although DMD is currently incurable, there is vast research into the disease. Genetic therapy with aims to reintroduce dystrophin back into cells looks promising; however, this research area does not come without its challenges and extensive work still needs to be done before this therapy will be available.

Currently, the only available treatments are physical therapy and pharmacological intervention using steroids. The use of physical therapy is controversial as some believe it is critical to help retain normal muscle function but some believe it can worsen the damage in the muscles. Administration of steroids (Prednisolone) is the most effective treatment; it can improve and it certainly slows down the progression of the disease, leading to a 
further 2-4 years of mobility for some sufferers. However, steroid use can include side effects, some patients are resistant to steroid treatment and the halt in disease progression is only temporary for all sufferers.

An under-researched area relating to Muscular Dystrophies is the increased incidence of mood disorders. We hypothesise that, some sufferers of the disease may also have depression, which goes undiagnosed, due to the physical symptoms of the disease masking the signs of depression. These symptoms include apathy, fatigue, low mood and sleep disturbances. For example, fatigue worsens muscle symptoms by increasing the apathy of the patients and reducing their mobility, suggesting that the emotional status of the patient directly interacts with the rate of progression of the disease.

The "monoamine theory of depression" describes an imbalance of chemicals, called neurotransmitters, in the brain, and their association with altered mood. Imbalance of neurotransmitters can lead to depression, anxiety and other physiological disorders. However, another theory, states that the immune system can cause depression, leading to the "macrophage theory of depression", named after the immune cell central to this effect. We hypothesise that both theories may be relevant in DMD.

Co-morbidity is when two or more disorders exist, sometimes by coincidence, sometimes one causing the other. Co-morbidity is extensively seen between physical disease and depression, and more so inflammatory disease and depression. One explanation for this is that pro-inflammatory immune cells are dysregulated in diseases such as arthritis, irritable bowel syndrome and cystic fibrosis. One interesting study demonstrates this clearly, depression in patients who have either inflammatory rheumatoid arthritis or noninflammatory osteoarthritis differ significantly, even though both types of arthritis have the same degree of disability and pain. A higher percentage of patients in the inflammatory rheumatoid arthritis group were found to have depression. If inflammation didn't contribute to depression in these cases, you would expect both groups to have the same prevalence of depression due to the comparative disability of the arthritis they have. We hypothesise that in a subset of DMD sufferers, the immune response may contribute to mood disorders.

Amitriptyline was one of the first drugs to be developed and prescribed as an antidepressant. It mediates the neurotransmitters, namely serotonin, involved in the pathogenesis of depression and anxiety. However, due to its chemical structure, amitriptyline has many different targets both in the brain and elsewhere in the body. Newer and more specific antidepressants are now available and amitriptyline is prescribed less frequently as a result, but new uses for amitriptyline have continued to emerge at different targets in the body. Amitriptyline is now prescribed for a variety of psychological disorders, such as anxiety attacks, attention deficit hyperactivity disorder, posttraumatic stress disorder and insomnia. It is a painkiller, an anti-oxidant and has anti-inflammatory properties. It is prescribed in low doses for cystic fibrosis, irritable bowel syndrome and chronic pain states such as neu- 
ropathic pain. The mechanisms of action for these effects are largely unknown. In various experimental models of disease, amitriptyline has been effective in reducing inflammation in different tissues. It is thought that this effect is due to its ability to modulate components of the immune system including the pro-inflammatory cytokines. If amitriptyline can mediate neurotransmitters and immune cells, it may be useful in DMD patients who suffer from depression, whether it's caused by a chemical imbalance or a dysregulated immune system.

We hypothesise that amitriptyline may be beneficial to the sufferers of DMD due to the depressive-like symptoms, the inflammatory response in muscle and a possible correlation and interaction of these symptoms of the disease.

In this study we used dystrophin-deficient mice, a mouse model of DMD, to first establish if the mouse exhibited depressive symptoms and whether amitriptyline could reverse this effect and secondly to investigate if amitriptyline works as an anti-inflammatory to slow the progression of the disease in skeletal muscle.

\section{Methods}

We first compared mice with dystrophin deficiency (Mdx mice) to healthy mice. Then we took a second set of Mdx mice and divided them into two groups, untreated and amitriptyline-treated and repeated the experiments.

To investigate whether the mice had mood disturbances, we used two established behavioural tests. In an open field test, exploratory behaviour was scored as mice entering the centre of the brightly lit arena as opposed to mice staying around the edges. Both exploring and hiding are natural behaviours of mice and the measure of exploratory behaviour correlates to anxiety. Ambulation was also analysed to compare the locomotor activity of Mdx mice compared to healthy mice, although it is reported that Mdx mice of this age (7 weeks) have no functional deficits in their muscles making them as active as healthy mice. Mdx mice and healthy mice then undertook a tail suspension test, which assesses behaviour in an inescapable environment. Depressed mice "accept their fate" they do not try to escape whereas non-depressed mice will try to escape.

To assess the anti-inflammatory effect of amitriptyline, skeletal muscle histology was undertaken. Skeletal muscle from the forelimb was dissected out, stained to differentiate between muscle and inflammatory cells and then visualised using bright-field microscopy. In these sections, infiltration of the muscle by activated immune cells can be scored by the distinct episodes of inflammatory cells invading the tissue and the total area of these inflammations per image. 


\section{Results}

Mdx mice spent less time exploring the open space in the open field behavioural test than normal mice. This indicates that the Mdx mice are more anxious than healthy mice. We then repeated the experiment with Mdx mice treated with amitriptyline compared to untreated $\mathrm{Mdx}$ mice. Amitriptyline-treated mice spent more time exploring compared to untreated mice - indicating that amitriptyline is effective in reducing anxiety in $\mathrm{Mdx}$ mice. When comparing ambulation in the open field test we found $\mathrm{Mdx}$ mice were not less active than healthy mice, indicating their muscles had no functional deficits at this age, but did not explore the open area as the healthy mice did. These control experiments further validate the behavioural test results, indicating that the anti-depressant and anxiolytic effects of amitriptyline are not obscured by alterations in mouse locomotion due to the disease.

Similarly, Mdx mice spent less time trying to escape the tail suspension test than healthy mice indicating depressive like symptoms in the mouse model. When treated with amitriptyline, Mdx mice spent significantly more time trying to escape than untreated mice, illustrating the anti-depressant effects of amitriptyline.

Inflammatory events were counted for both frequency and area of events per image of each section of muscle of both Mdx- and healthy mice. Mdx mice had a significantly higher frequency and area of inflammation than healthy mice which is consistent with previous studies. When Mdx mice were treated with amitriptyline, the frequency of inflammation and the area of inflammation were reduced in the Mdx mouse. Inflammatory events were defined visually, in line with previous histological studies of Mdx muscle. These results indicate that amitriptyline may be useful in reducing the inflammatory response in dystrophin deficiency induced inflammation. However, this warrants further study to establish which cells from the inflammatory response are involved and which cells are reduced with the treatment of amitriptyline.

\section{Conclusion and Summary of Results}

The Mdx mouse model of DMD exhibits more anxiety and depression than their control healthy mice in two behavioural tests. When we treated these Mdx mice with amitriptyline and compared them to untreated Mdx mice, we found that amitriptyline reduced depression and anxiety behaviours in the mice.

Ambulation is an important factor when comparing Mdx mice to healthy mice; if these mice suffered from loss of mobility, the test results would be obscured and not valid. Control experiments investigating ambulation show both the distance and time spent walking during the open field test were comparable between the strains, indicating that this is 

Dystrophy?

likely to be a true effect of amitriptyline.

Mdx mice had significantly more inflammation than healthy controls, as indicated by the frequency and area of inflammation. However, amitriptyline was effective in reducing both the frequency and area of inflammation in the Mdx mice skeletal muscle.

These results suggest that amitriptyline may be useful in providing relief from both psychological and physical symptoms of the disease and thus providing a better quality of life for patients with DMD. We are undertaking further investigations regarding both the type of chemical disturbances in the brain underlying the pathogenesis of the mood disturbances and also which type of immune cells are infiltrating the skeletal muscle tissue and by what mechanisms amitriptyline is working here. This study will further help us understand how amitriptyline works and may lead to a new use for an old drug.

With thanks to Dr. Yilmazer-Hanke, my supervisor Dr. Dervla O'Malley (Dept. of Physiology), my colleagues in the Anatomy \& Neuroscience Department and in the Physiology department. I would like to acknowledge Muscular Dystrophy Ireland who provided the funding for this project. 